\title{
MEASUREMENT OF THE MECHANICAL BEHAVIOUR OF THE LHC BEAM SCREEN DURING A QUENCH
}

\author{
J. Martinez-Darve, K. Artoos, EST-ME, CERN, Geneva, Switzerland \\ P. Cruikshank, N. Kos, C. Rathjen, LHC-VAC, CERN, Geneva, Switzerland
}

\section{Abstract}

The LHC (Large Hadron Collider) beam vacuum system will be equipped with a so-called beam screen. This screen, operating between $5 \mathrm{~K}$ and $20 \mathrm{~K}$, is coaxially placed inside the beam pipe to reduce the beam induced heat load to the superconducting coil. The racetrack shaped stainless steel screen is perforated to provide vacuum pumping and has an inner layer of $50 \mu \mathrm{m}$ high purity copper to limit the machine impedance.

The beam screen needs to resist the induced Lorentz forces during a resistive transition (quench) of the magnet. This paper reports the measurements carried out on a 15.6 metre long screen tested in aperture 1 of the twin aperture prototype LHC main dipole magnet MBP2O1. The quench induced deformation of the beam screen cross-section, the angular displacement and the temperature increase after a quench have been measured with instrumentation developed for this test. Optical transducers, not influenced by the magnetic field, allowed measuring the deformation of the cross section with high resolution and precision. This gave an insight into the mechanisms that govern the behaviour of the beam screen during a quench. Finally, some "quench rings" positioned inside the beam screen were used to check the thickness of the beam screen wall where plastification would occur.

\section{INTRODUCTION}

A 15.6 metre long, racetrack shaped beam screen with cooling tubes and pumping slots was inserted in aperture 1 of the prototype magnet MBP2O1. The UNS21904 stainless steel beam screen has a $46 \mathrm{~mm}$ outer diameter and $1 \mathrm{~mm}$ thickness [1]. The beam screen was supported by spring supports every $1519 \mathrm{~mm}$ in the $50 \mathrm{~mm}$ inner diameter cold bore tube. The internal surface of the beam screen is covered with a $50 \pm 5 \mu \mathrm{m}$ thick copper layer, whose RRR varied between 50 and 70 around the circumference. Eddy currents will be induced in this low resistance copper layer during a magnet resistive transition, resulting in induced Lorentz forces and an increase of the beam screen temperature.

With the temperature of the magnet fixed at $1.8 \mathrm{~K}$, the temperature of the beam screen was controlled to remain between 6 and $30 \mathrm{~K}$ by means of a heater and a flow of helium gas in one of the two cooling tubes of the beam screen. The magnet was quenched at different magnetic fields up to nominal $(8.3 \mathrm{~T})$ and ultimate $(9 \mathrm{~T})$ field in the aperture.

\section{INSTRUMENTATION}

The instrumentation used during the test consisted of five displacement transducers based on strain gauges measuring the horizontal and the vertical deformation of the beam screen cross-section during a quench. Two optical displacement transducers based on the Moiré effect measured the horizontal deformation [2, 3, 4, 5, 6]. Four free standing $32 \mathrm{~mm}$ inner diameter rings in P506 stainless steel with a $50 \mu \mathrm{m}$ copper layer (RRR 50) on their inner surface were placed inside the beam screen. These so-called "quench rings" with different wall thicknesses $(0.15,0.19,0.26$ and $0.29 \mathrm{~mm})$ were used to study the previously estimated thickness for which plastic deformation would occur.

A torsion transducer based on strain gauges was mounted on the free end of the beam screen. The other end was a fixed point. The transducer measured the quench induced beam screen twist.

Two full range temperature gauges were mounted on the beam screen.

A Labview ${ }^{\circledR}$ program managed the triggered acquisition, the data storage, processing and the display of the results.

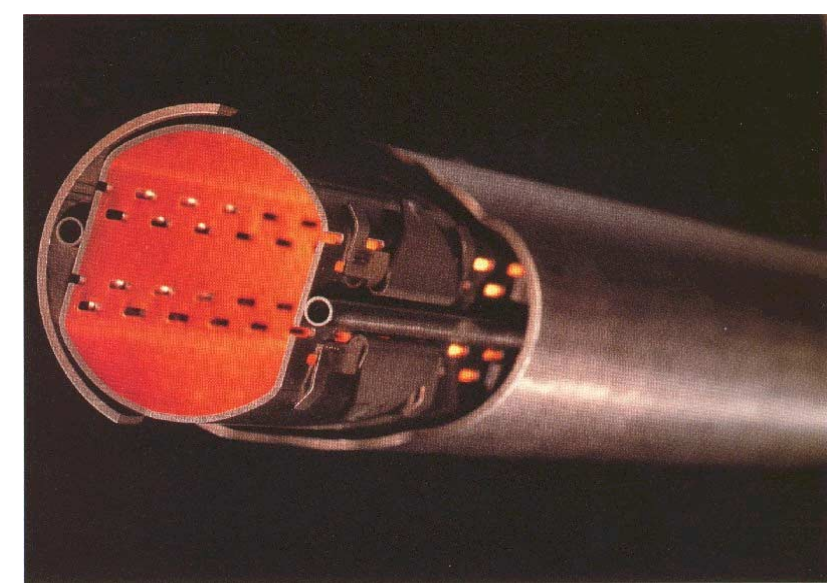

Figure 1. The beam screen with pumping slots, cooling tubes and support inside a cold bore tube

\section{RESULTS}

\subsection{Deformation of the cross-section of the beam screen during the quench}

The quench induced deformation of the beam screen cross-section was measured by displacement transducers based on strain gauges and an optical method. For both methods a linear proportionality of the deformation versus $\mathrm{BdB} / \mathrm{dt}$ ( $\mathrm{B}$ being the magnetic induction) could be determined. The slope of this linear part was found to be reproducible for different quenches. The maximum deformation during the quench could not be read directly 
with the strain gauges due to the high level of noise during the quench and a zero offset at the start of the quench. The measurements with the optical transducers were noise free and allowed to determine directly a maximum horizontal deformation of $300 \mu \mathrm{m}$ of the beam screen cross-section during a quench at nominal field (figure 2).

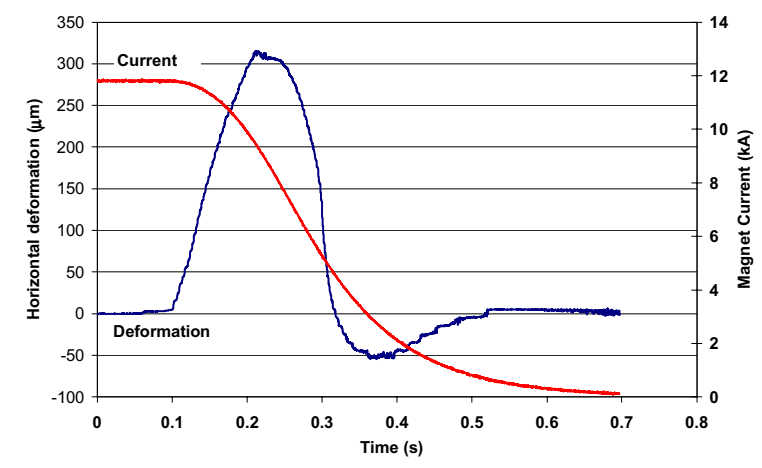

Figure 2. Horizontal beam screen deformation (optical) and magnet current during a quench

The measured deformation plotted against $B d B / d t$ (figure 3) is almost linear between the start of a quench and the maximum (absolute value). This confirmed a previous model [7].

The rate of deformation versus $B d B / d t$ changed slope at a certain point after the maximum deformation for quenches at $6.4 \mathrm{~T}$ and higher. The deformation decreased down to negative values. This means that the beam screen was compressed in the horizontal direction. Later tests showed that the contact of the beam screen to the cold bore tube caused the negative deformation that was measured during the quench tests. A contact between the beam screen and the cold bore tube can only be explained by unbalanced Lorentz forces on the beam screen during a quench. Since the net sum of the induced eddy currents is zero, unbalanced Lorentz forces must be due to an additional superimposed current. A possible explanation for the source of this current was given recently $[6,8]$.

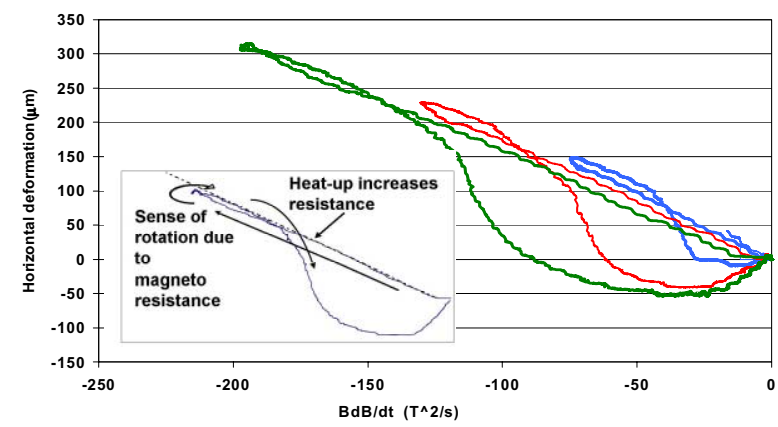

Figure 3. Horizontal deformation measured by the optical transducers versus $B d B / d t$

The displacement versus $B d B / d t$ curves made a clockwise turn at the maximum displacement for quenches between $6.4 \mathrm{~T}$ and $8.3 \mathrm{~T}$. This indicates that the magneto resistance of the copper layer is decreasing, due to the decreasing magnetic field, faster than the increase of the electrical resistance due to the rising temperature. The small area between the increasing and decreasing part of the curve (up to the point of contact with the cold bore tube) shows that the decreasing magneto resistance is well compensated by the thermal increase of the resistance. It is hence a good approach to assume constant resistance of the copper layer during the quench.

To allow a comparison between the two measurement methods, the rate of the deformation versus $B d B / d t$ was calculated from the linear part of the measurements for the strain gauge based transducers and the optical transducers. With this rate and the measured $B d B / d t$ the maximum deformation was determined for both methods and can be compared with the maximum displacement directly obtained from the optical transducers (figure 4). The error margins were estimated by comparison of the results obtained from quenches at the same quench current and the same beam screen temperature.

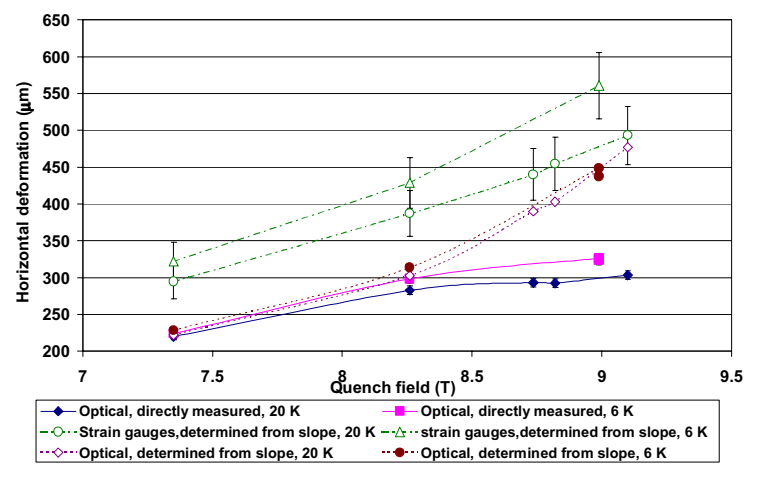

Figure 4. Maximum horizontal deformation

The average ratio of the vertical to the horizontal beam screen deformation measured with the strain gauge transducers was 0.97 , i.e. the computed value [3] [9].

The maximum deformation directly measured by the optical transducers is probably the most accurate result because of the inherent precision of the method, the noise free signal and the very good repeatability. The maximum deformation determined from the rate of deformation versus $B d B / d t$ is a less precise method because of the nonlinear parts (for the optical transducers) and the random influence from the noise (for the strain gauges based transducers).

\subsection{Deformation of the quench rings}

Table 1: "Quench rings" plastic horizontal deformation

\begin{tabular}{|c|c|c|c|c|}
\hline $\begin{array}{c}\text { Steel wall thickness } \\
(\mathrm{mm})\end{array}$ & 0.15 & 0.19 & 0.26 & 0.29 \\
\hline $\begin{array}{c}\text { Plastic horizontal } \\
\text { deformation (mm) }\end{array}$ & 3.20 & 1.20 & 0.40 & 0.20 \\
\hline
\end{tabular}

The four rings were removed after the test and the diameter was measured. All four rings were plastically deformed. The measurement also validated the method used to estimate the wall thickness of the quench rings where plastification occurs; the order of magnitude of 
results from the test and the F.E. model is the same [5].

\subsection{Angular displacement of the beam screen during a quench}

The highest relative angular displacement between the beam screen and the magnet cold mass measured during a quench was $7 \mathrm{mrad}$. This value is much smaller than the 35 mrad measured during the quench tests of a square cross section prototype [10]. It is known than a nonuniformity of the copper thickness can create unbalanced eddy currents resulting in a torque that rotates the beam screen. The current co-laminated copper layer is much more homogeneous than the former electro-deposited one and this consequently reduces the torque. The twist measured during the test was mainly due to the deformation of the magnet cold mass because of the pressure increase inside. The maximum twist does not happen at the maximum absolute value of $B d B / d t$ but later. No permanent twist of the beam screen has been observed.

\subsection{Temperature increase during the quench}

The temperatures were measured by two sensors on the outside of the stainless steel beam screen wall. They were located at $2 \mathrm{~m}$ from each end of the beam screen. The induced currents during a quench flow however only in the copper layer. The energy that is dissipated will increase the temperature of the copper layer and it is only the temperature after homogenisation over the beam screen wall that was measured. A model [7] indicated that the temperature is homogeneous five seconds after the quench for a thermally isolated beam screen. The measured temperature increased during the quench and continued to increase 3 seconds after the quench. From this moment the temperature near to the cooling tube inlet decreased since the screen cooling system remained open. The temperature on the other side continued to increase to reach the temperature of the cold mass after a quench by conduction through the cooling tube.

The average maximum temperature of the beam screen wall 3 seconds after a quench at nominal field was $22.9 \mathrm{~K}$ (for an initial beam screen temperature of $6.1 \mathrm{~K}$ ) and 26.3 $\mathrm{K}$ (for an initial beam screen temperature equal to 19.2 $\mathrm{K})$. Those temperatures were used as the homogenised temperatures to estimate the maximum temperatures of the copper wall during a quench. This was done by considering the conservation of energy during the temperature homogenisation over an adiabatic beam screen [5]. For a quench at nominal field, maximum copper layer temperatures of $58 \mathrm{~K}$ and $56 \mathrm{~K}$ were calculated this way for an initial beam screen temperature of respectively $6.1 \mathrm{~K}$ and $19.2 \mathrm{~K}$.

\section{CONCLUSION}

The first quench induced beam screen deformation measurements were carried out on a full-length prototype racetrack shaped beam screen in MBP2O1. Quenches were made from $2.1 \mathrm{~T}$ to $9.1 \mathrm{~T}$. Two new measurement methods were successfully used: a deformation measurement based on optical gratings and the so called quench rings.

The optical transducers allowed a more precise and direct measurement, which showed that the results from the strain gauge based transducers were too high. A horizontal deformation of $0.30 \mathrm{~mm}$ at $200 \mathrm{~T}^{2} / \mathrm{s}$ was measured with an initial beam screen temperature of 20 $\mathrm{K}$. There was no permanent deformation of the beam screen after the quench tests. More subtle effects like the change of the resistance of the copper layer due to temperature and magneto resistance could be studied.

The optical transducers also showed that the beam screen was pulled against the cold bore tube for quenches higher than $6.4 \mathrm{~T}$ because of additional induced currents.

The quench rings permitted to show that plastic deformation will occur during quenches below a certain beam screen wall thickness.

The highest temperature reached by the beam screen wall after a quench depends on the temperature of the cold mass after the quench and the heat flow through the walls of the cooling tubes. The maximum temperature reached by the copper layer during the quench was calculated from the temperature measurements on the outside beam screen wall before and after the quench. A maximum copper layer temperature of $58 \mathrm{~K}$ and $56 \mathrm{~K}$ respectively was calculated for an initial beam screen temperature of $6.1 \mathrm{~K}$ and $19.2 \mathrm{~K}$.

\section{ACKNOWLEDGEMENTS}

The authors wish to express their thanks to the people that participated to the preparation of the beam screen test and the team responsible for the magnet test bench.

\section{REFERENCES}

\section{[1] CERN drawing LHCVSSBA0003}

[2] C. Rathjen; Strain gauge based displacement transducer. Technical Note CERN EST-ESI/99-07.

[3] J. M-Darve; Ratio between the vertical and horizontal deformation of the cross-section of a LHC beam screen during quench. Technical Note CERN EST-ME/01-001.

[4] C. Rathjen; Optical displacement transducer for applications at cryogenic temperatures and high magnetic fields. Technical Note LHC-VAC/00-22.

[5] K. Artoos, J M-Darve, C. Rathjen; Beam screen quench test in a 15 metre long LHC dipole magnet. Technical Note EST-ME/2001-003.

[6] C. Rathjen; Currents in, Forces on and Deformations/Displacements of the LHC Beam Screen Expected during a Magnet Quench. PAC-2001, ID: TOAB005.

[7] C. Rathjen; A model to calculate LHC beam screen deformation in a dipole field during quench. Technical Note LHC-VAC/00-21.

[8] A. Siemko, F. Caspers, CERN. Priv. communication.

[9] R.Valbuena, CERN. Private communication.

[10] K. Artoos, P. Cruikshank, N Kos; Mechanical and thermal measurements on a $11 \mathrm{~m}$ long beam screen in the LHC Magnet Test String during the RUN 3A. LHC Project Note 178 